\title{
Distribuição, dominância e estrutura de tamanhos da assembleia de peixes da lagoa Mangueira, sul do Brasil
}

\author{
Luiz G. S. Artioli, João P. Vieira, Alexandre M. Garcia \& Marlise de A. Bemvenuti
}

Laboratório de Ictiologia, Instituto de Oceanografia, Universidade Federal do Rio Grande, Caixa Postal 474, 96201-900 Rio Grande, RS. (lgartioli@hotmail.com)

\begin{abstract}
Distribution, dominance and sizes structure of the fish assemblage in the Mangueira lake, southern Brazil. This study presents the composition of species, the distribution and dominance patterns, and the structure of sizes of the assemblage of fish in distinct habitats (littoral, deeper and near shore limnetic zone) of the Mangueira lake, in the southern Brazil. Samples were taken in the north, south and central regions of the lake from 2001 to 2007 using a multi-gear approach (gillnet, cast net, beach seine, dip net and bottom trawling). A total of 52 species were identified, 46 in the littoral zone, 33 in the near shore limnetic zone and 26 in the deep zone, distributed in 17 families, of the which, Characidae, Cichlidae, Loricariidae, and Atherinopsidae were the most diverse. The dominant species summed up $91.1 \%, 92.9 \%$ and $82.7 \%$ of the all individuals caught in the littoral, deeper and limnetic zone, respectively. The littoral zone was comprised of small fishes, nearly $70 \%$ between 25 and $50 \mathrm{TL} \mathrm{mm}$. The dominance patterns showed that, in this zone, $19,5 \%$ of the species were dominant, although four of those also prevailed in the other zones. In the deep zone, 7,6\% of the captured species were dominant, with similar sizes to the littoral zone. For the other species, the sizes resemble to the near shore limnetic zone. The near shore limnetic zone was comprised of larger fishes, the major part between 150 and $200 \mathrm{TL}$ mm, such as the great characins, the toothless characin and the silverside fishes. A larger difference was observed in the composition of species between the littoral and limnetic zones, with the species Jenynsia multidentata, Bryconamericus iheringii, Hyphessobrycon luetkenii and Gymnogeophagus gymnogenys dominating exclusively in the littoral, and the species Oligosarcus jenynsii, Cyphocharax voga, Odontesthes perugiae and Astyanax fasciatus dominating exclusively the near shore limnetic zone. The littoral zone presented differences in the composition and dominance of the species among the portions north and south of the lake. The 52 species registered in this study represent a high diversity of fishes in Mangueira lake, specially when compared to other coastal lakes of RS. Differentiated patterns of composition and abundance of species seem to reflect characteristics different from the coastal habitats sampled.
\end{abstract}

KEYWORDS. Coastal lakes, biodiversity, ichthyofauna.

RESUMO. Este estudo apresenta a composição de espécies, os padrões de distribuição e dominância, e a estrutura de tamanhos da assembleia de peixes em diferentes unidades de hábitat (zonas litoral, profunda e limnética costeira) da lagoa Mangueira, no extremo sul do Brasil. As amostras foram obtidas no norte, centro e sul da lagoa, entre os anos de 2001 e 2007 utilizando-se de diversos amostradores (rede de emalhe, tarrafa, rede de arrasto de praia, puçá e rede de arrasto de fundo). Um total de 52 espécies foi identificado, 46 na zona litoral, 33 na limnética costeira e 26 na profunda, distribuídas em 17 famílias, das quais, Characidae, Cichlidae, Loricariidae e Atherinopsidae foram as mais diversas. As espécies dominantes somaram $91,1 \%, 92,9 \%$ e 82,7 \% dos indivíduos capturados nas zonas litoral, profunda e limnética costeira respectivamente. A zona litoral é constituída de pequenos peixes, quase $70 \%$ entre 25 e $50 \mathrm{~mm}$ de comprimento. Os padrões de dominância mostraram que, nesta zona, $19,5 \%$ das espécies foram dominantes, embora quatro dessas também predominaram nas demais zonas. Na zona profunda, 7,6 \% das espécies capturadas foram dominantes, com tamanhos semelhantes aos da zona litoral. Para as demais espécies, os tamanhos se assemelham aos da zona limnética costeira. A zona limnética costeira é constituída de peixes de maior porte, a maioria entre 150 e $200 \mathrm{~mm}$ de comprimento total, tais como os grandes caracídeos, o birú e os peixes-rei. Observou-se uma maior diferença na composição de espécies entre as zonas litoral e limnética costeira, com as espécies Jenynsia multidentata, Bryconamericus iheringii, Hyphessobrycon luetkenii e Gymnogeophagus gymnogenys dominando exclusivamente no litoral, e as espécies Oligosarcus jenynsii, Cyphocharax voga, Odontesthes perugiae e Astyanax fasciatus dominando exclusivamente a zona limnética costeira. A zona litoral apresentou diferenças na composição e dominância das espécies entre as porções norte e sul da lagoa. As 52 espécies registradas neste estudo representam uma alta riqueza de peixes na lagoa Mangueira, sobretudo quando comparada a outras lagoas costeiras do RS. Padrões diferenciados de composição e abundância de espécies parecem refletir características distintas dos habitats litorâneos amostrados.

PALAVRAS-CHAVE. Lagoas costeiras, biodiversidade, ictiofauna.

Lagoas costeiras ocorrem ao longo de toda a costa brasileira, constituindo-se em um dos conjuntos de ecossistemas aquáticos continentais mais numerosos do país (Esteves, 1998). No estado do Rio Grande do Sul, a planície lagunar adjacente à costa possui inúmeras lagoas que recebem a drenagem natural da maior parte do sistema hidrográfico do Estado (Delaney, 1965). Destacam-se, além das duas grandes lagoas, Patos e Mirim, a lagoa Mangueira, que na sua porção norte, juntamente com diversas lagoas de menor porte, integra o sistema hidrográfico do Taim, onde localiza-se a Estação Ecológica do Taim (EETaim).

Embora os peixes usualmente representem um importante componente biológico em lagoas e banhados de água doce (DeAngelis et al., 2005; CARPEnTER et al., 1985; Bruton \& Merron, 1990; Welcomme, 1979), a ictiofauna desses ecossistemas tem sido pouco estudada. Os esparsos estudos sobre a ictiofauna da lagoa Mangueira referem-se à sua porção norte, no contexto da EETaim (BucKup, 1981; BuCKUP \& MALABARBA, 1983; ReIs, 1983; Grosser et al., 1994; Moresco \& BemVenuti, 2005; Piedras \& Pouey, 2005; Garcia et al., 2006).

O presente trabalho investiga possíveis diferenças em termos de padrões de composição de espécies, padrões de dominância, variação espacial e temporal e de estrutura de tamanhos da assembleia de peixes presentes em distintas unidades de hábitat (zonas litoral, profunda e limnética) da lagoa Mangueira. 


\section{MATERIAL E MÉTODOS}

Área de estudo. A lagoa Mangueira localiza-se na região sul do Estado do Rio Grande do Sul, entre as dunas que separam o município de Santa Vitória do Palmar e o

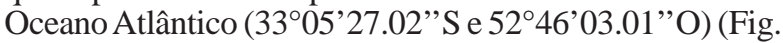
1). Do ponto de vista geológico é uma lagoa costeira bastante jovem (cerca de 5.000 anos), formada a partir de regressões e transgressões marinhas através de processos de erosão e deposição de areia juntamente com a ação eólica (SCHÄFER, 1984; TOMAZELLI \& VILLWOCK, 2005). Possui $92 \mathrm{~km}$ de comprimento, cerca de dois a seis $\mathrm{km}$ de largura e profundidade média oscilando entre 1,5 e seis metros, o que resulta em um extenso espelho d'água (cerca de $820 \mathrm{~km}^{2}$ ) e volume aproximado de setecentos milhões de metros cúbicos de água doce (Delaney, 1965).

Coleta de dados. Zona Litoral. Foram realizadas amostragens mensais de março de 2004 a fevereiro de 2005, e com periodicidade variada até fevereiro de 2007 (ver descrição em zona limnética) em dois pontos fixos, ao norte (Fazenda Santa Marta) e ao sul (Fazenda Porteira de Ferro), e duas campanhas de amostragens, em agosto de 2006 e janeiro de 2007, na parte central leste e oeste (Fig. 1), totalizando 190 arrastos em 22 dias de trabalho. O ponto norte consiste de uma praia arenosa, de vegetação aquática esparsa, pouca profundidade e baixo declive, em área legalmente protegida, integrante da EETaim. O ponto sul de amostragem constituiu-se de um canal de irrigação onde tanto a profundidade quanto o declive são bem mais acentuados, contudo, com uma intensa colonização por macrófitas aquáticas submersas.

As amostras foram obtidas com rede de arrasto de praia tipo trawl, com $9 \mathrm{~m}$ de largura e 2,4 $\mathrm{m}$ de altura, malha de $13 \mathrm{~mm}$ nas asas e $5 \mathrm{~mm}$ no saco da rede, que foi utilizada em profundidades menores que $1,5 \mathrm{~m}$, em diferentes habitats (VIEIRA et al., 2006); e puçá de malha de $5 \mathrm{~mm}$, empregado em profundidades menores que um metro. O esforço de coleta foi diferente entre os dois amostradores, com predomínio da rede de arrasto de praia. Os peixes coletados com puçá foram utilizados apenas para compor a lista de espécies.

Zona profunda. A parte central da lagoa teve a zona profunda amostrada em três expedições (Fig. 1). A primeira em agosto de 2006 e a segunda e terceira em janeiro e fevereiro de 2007, respectivamente, totalizando 23 arrastos em três dias de trabalho.

Foram efetuados arrastos de fundo (de dois a oito metros de profundidade) de 10 minutos, utilizando-se uma rede do tipo wing trawl, com tralha superior de 8,62 $\mathrm{m}$ de comprimento, tralha inferior de $10,43 \mathrm{~m}$ de comprimento, peso do chumbo de 27 unidades de 60 gramas (1,62 kg), malhas de $13 \mathrm{~mm}$ (fio 210/09) nas mangas e barriga, e malha de $5 \mathrm{~mm}$ (fio 210/12) no saco. Foram utilizadas portas de madeira vazada $(70 \mathrm{~cm} \mathrm{x} 42 \mathrm{~cm}) \mathrm{e}$ peso de $9,3 \mathrm{~kg}$ (VIEIRA et al., 2006). Também foi utilizada, com menor esforço amostral, rede do tipo beam trawl, com boca de $1 \times 0,5 \mathrm{~m}$, malha de $13 \mathrm{~mm}$ no corpo e três $\mathrm{mm}$ no saco da rede (GARCIA \& VIEIRA, 1997).

Zona limnética. Foram realizadas amostragens de periodicidade variada (março, julho e dezembro de 2001; abril, julho e novembro de 2002; fevereiro, julho, outubro e dezembro de 2003; março, maio, julho, setembro e dezembro de 2004; dezembro de 2005; março, junho e setembro de 2006 e fevereiro de 2007) com rede de emalhe com diferentes tamanhos de malha $(15,20,30,35 \mathrm{~mm}$ entre nós adjacentes). Cada malha teve um pano $2 \times 2 \mathrm{~m}$, totalizando um painel com oito metros de comprimento por dois metros de altura. Em cada local de amostragem foram colocadas duas redes de quatro painéis, em distâncias entre 100 e 200 metros da margem, no início e no fim da tarde no norte e sul da lagoa, respectivamente, e retiradas no dia seguinte, permanecendo em média 22 horas no norte e 15 horas no sul em cada amostragem. Em profundidades maiores que um metro, nas regiões abertas e canais naturais e artificiais, foi empregada tarrafa de $0,5 \mathrm{~m}$ de diâmetro e malha de $15 \mathrm{~mm}$ entrenós adjacentes. O esforço amostral foi diferente entre os dois amostradores, com predomínio da rede de emalhe. Os peixes coletados com tarrafa foram utilizados apenas para compor a lista de espécies.

Processamento e análise dos dados. A identificação das espécies foi feita com auxílio de bibliografia (REIS, 1983; Buckup \& Reis, 1997; Rodrigues \& Bemvenuti, 1997; Bemvenuti, 2002; Bemvenuti \& Rodrigues, 2002; Bemvenuti \& Moresco, 2005) e de especialistas. As espécies foram identificadas por amostrador e foi verificada a contribuição percentual das mesmas nas diferentes unidades taxonômicas.

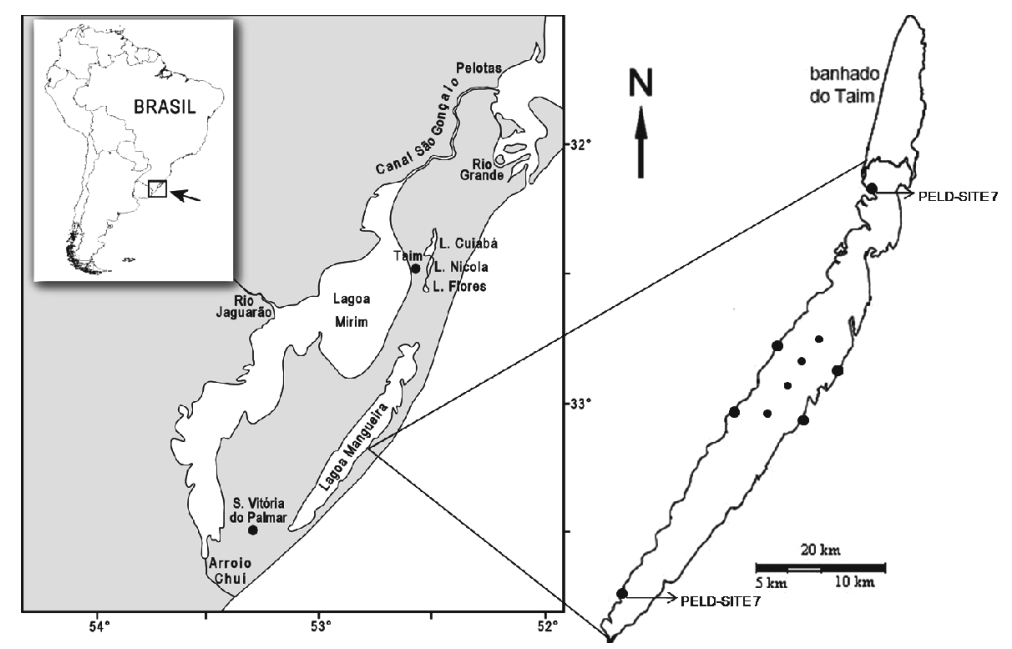

Figura 1. Mapa da região sul do Brasil com a localização da lagoa Mangueira indicando os pontos de amostragem no âmbito do programa PELD-CNpq site-7 e na parte central de lagoa (círculos pretos maiores correspondem a zona litoral e os menores a zona profunda). 
Padrões de distribuição e dominância. Para caracterizar as unidades de habitat em termos de presença de espécies, as amostras de arrasto de praia e puçá foram agrupadas, caracterizando a assembleia de peixes da zona litoral, assim como, as amostras de arrasto de fundo e beam trawl caracterizaram a assembleia de fundo e as amostras de rede de espera e tarrafa a assembleia da zona limnética.

Para as análises de abundância e frequência relativas por unidade de hábitat foram utilizados apenas os amostradores com esforço relevante onde foi utilizada a CPUE (captura por unidade de esforço), a FO \% (frequência de ocorrência), onde: FO \% é a razão entre o número de vezes em que a espécie ocorreu sobre o número total de amostras multiplicado por 100; e o PN \% (percentual numérico) de cada espécie, sendo que o PN \% é a razão entre o número de indivíduos de uma espécie sobre o número total de indivíduos coletados multiplicado por 100. Para os diferentes amostradores, a CPUE de cada espécie foi calculada pelo número total de indivíduos capturados divididos pelo esforço de captura. O esforço para os arrastos de praia e de fundo é igual ao número total de arrastos e, para a rede de emalhe, o esforço é igual ao número total de horas que rede esteve dentro d'água.

Como a natureza do esforço foi diferente entre os amostradores, para verificação da importância relativa de cada espécie em cada unidade de hábitat, assim como o seu padrão de dominância, optou-se por utilizar uma combinação de PN \% (baseado em CPUE) e FO \%, onde os valores de $\mathrm{PN} \%$ e FO \% foram comparados com suas respectivas médias ( $\mu \mathrm{PN} \%$ e $\mu \mathrm{FO} \%)$. As espécies foram assim classificadas: abundante e frequente (PN \% $\geq \mu \mathrm{PN}$ $\%, \mathrm{FO} \% \geq \mu \mathrm{FO} \%$ ); abundante e não frequente (PN $\% \geq$ $\mu \mathrm{PN} \%, \mathrm{FO} \%<\mu \mathrm{FO} \%$ ); não abundante e frequente (PN $\%<\mu \mathrm{PN} \%, \mathrm{FO} \% \geq \mu \mathrm{FO} \%)$ e presente $(\mathrm{PN} \%<\mu \mathrm{PN} \%$, FO $\%<\mu \mathrm{FO} \%)$. As espécies identificadas como abundantes e frequentes foram consideradas como dominantes, tanto entre diferentes amostradores quanto entre unidades de hábitat.

Para a análise de similaridade de presença/ausência de espécies entre as unidades de hábitat foi utilizado o coeficiente de Jaccard $(\mathrm{Cj})$ conforme descrito em MAGURRAN (1988) $[\mathrm{Cj}=\mathrm{j} /(\mathrm{a}+\mathrm{b}-\mathrm{j})$ onde: $\mathrm{j}=$ número de espécies comuns $\mathrm{a}$ ambos os habitats; $\mathrm{a}=$ número de espécies no hábitat $\mathrm{A}$ e $\mathrm{b}$ = número de espécies no hábitat $\mathrm{B}$ ]. Para a análise quantitativa, foi utilizado o percentual de similaridade $(\mathrm{P})$ conforme descrito em KREBS (1989) [P = Si mínimo (p1i, p2i) onde: $\mathrm{p} 1 \mathrm{i}=$ percentual da espécie $\mathrm{i}$ na $\operatorname{amostra} 1$ e p2 $\mathrm{i}=$ percentual da espécie i na amostra 2]. Para as análises de similaridade de presença/ausência entre os pontos de coleta da zona litoral ao norte e ao sul da lagoa (PELD-CNPq site7), também foram utilizadas as técnicas de agrupamento modo Q (Cluster - método normal) e de ordenação MDS (Non Metric Multidimensional Scaling) (GAUCH, 1982). A similaridade entre os atributos foi calculada pelo coeficiente de similaridade de Bray-Curtis, e os grupos foram formados pela média dos valores de similaridade dos grupos (Group average). Utilizou-se o programa estatístico PRIMER versão 6.1.9.

Estrutura de tamanho das assembleias. Para esta análise foram estabelecidas classes de comprimento total (CT) de cinco e $10 \mathrm{~mm}$. A variação da abundância por classe de CT foi baseada no cálculo de CPUE-CT (captura por unidade de esforço por classe de comprimento total) (VIEIRA, 2006) e transformada em CPUE-CT percentual. ACPUE-CT de cada espécie foi obtida por meio da razão entre a soma do fator de ponderação $(\mathrm{FP}=\mathrm{N} / \mathrm{n}$ onde $\mathrm{N}$ é o número de indivíduos capturados na amostra e n o número de indivíduos medidos) e o esforço ( $\mathrm{FP}(\mathrm{CT}) /$ esforço).

\section{RESULTADOS}

Foram identificadas 52 espécies de peixes distribuídas em 17 famílias e seis ordens. As famílias mais representativas em número de espécies $(\mathrm{S})$ foram Characidae $(S=16)$, Cichlidae $(S=7)$, Loricariidae $(S=6)$ e Atherinopsidae $(\mathrm{S}=5)$, contendo $65 \%$ das espécies. As ordens Characiformes e Siluriformes foram as mais representativas ( $S=20 ; S=15$ respectivamente), totalizando $67 \%$ das espécies capturadas (Tab. I).

Considerando-se todos os artefatos de pesca, um total de 33.070 indivíduos com biomassa total de $507,3 \mathrm{~kg}$ foi coletado entre março de 2001 e fevereiro de 2007. A análise dos padrões de dominância revelou que, na zona litoral, oito espécies, além dos juvenis de peixes-rei, foram dominantes e perfizeram mais de $90 \%$ dos indivíduos capturados. Na zona profunda apenas duas espécies foram dominantes e totalizaram $93 \%$ da captura. Na zona limnética seis espécies foram dominantes e totalizaram mais de $80 \%$ da captura (Tab. II).

Os valores de similaridade de Jaccard $(\mathrm{Cj})$ entre as assembleias estiveram próximos a 0,5 , resultando também em diferenças nos padrões de dominância entre elas. Apenas 13 espécies foram dominantes nas três assembleias, resultando em uma baixa similaridade em termos de abundância relativa $(\mathrm{P})$ entre os grupos (Zonas Profunda/Litoral $\mathrm{Cj}=0,49 ; \mathrm{P}=30,56$. Zonas Limnética/ Litoral $\mathrm{Cj}=0,51 ; \mathrm{P}=19,47$. Zonas Limnética/ Profunda $\mathrm{Cj}$ $=0,44 ; \mathrm{P}=2,03)$.

Quando analisada a matriz de dados de presença/ ausência das espécies coletadas nas porções norte e sul da zona litoral, entre março de 2004 e fevereiro de 2005, pelas técnicas de agrupamento (Cluster) e ordenamento (MDS) (Figs. 2, 3), verifica-se que praticamente todas as amostras do ponto sul formam um grupo (50\% de similaridade), o mesmo ocorrendo com as amostras do ponto norte (55\% de similaridade).

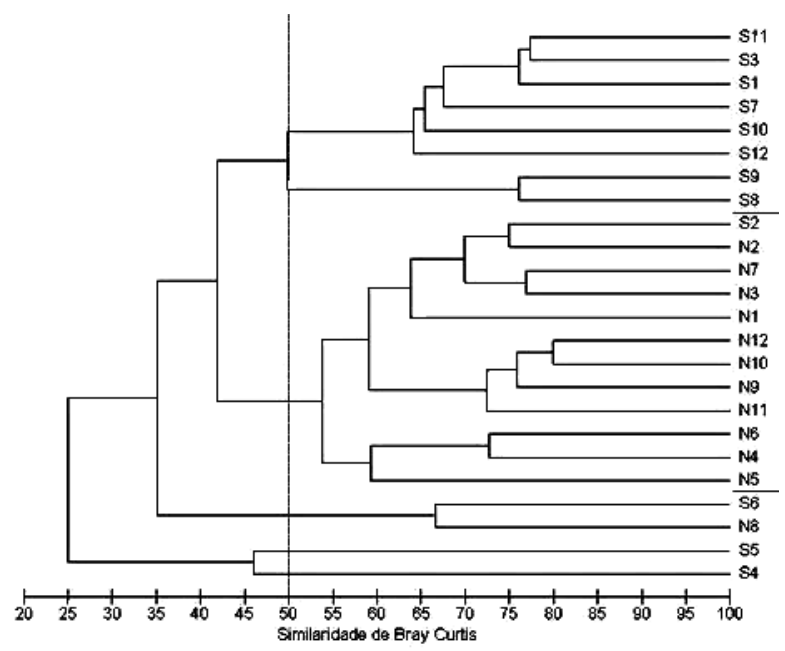

Figura 2. Dendrograma a partir dos dados de presença/ausência das espécies de peixes coletadas mensalmente entre março de 2004 e fevereiro de 2005 com rede de arrasto de praia, nos pontos de amostragem do programa PELD-site 7 na zona litoral da lagoa Mangueira ( $\mathrm{N}$, norte; $\mathrm{S}$, sul; 1 a 12 , amostras). 
Tabela I. Peixes coletados na lagoa Mangueira entre março de 2001 e fevereiro de 2007 com diferentes amostradores (1, emalhe; 2, tarrafa; 3, arrasto de praia; 4, puçá; 5, arrasto de fundo; x, representa presença nos respectivos amostradores).

\begin{tabular}{|c|c|c|c|c|c|c|}
\hline Taxons & Nome comum & 1 & 2 & 3 & 4 & 5 \\
\hline \multicolumn{7}{|l|}{ CLUPEIFORMES } \\
\hline \multicolumn{7}{|l|}{ Clupeidae } \\
\hline Platanichthys platana (Regan, 1917) & sardinha & & & $\mathrm{X}$ & $\mathrm{X}$ & $\mathrm{X}$ \\
\hline \multicolumn{7}{|l|}{ CHARACIFORMES } \\
\hline \multicolumn{7}{|l|}{ Curimatidae } \\
\hline Cyphocharax voga (Hensel, 1870) & biru & $\mathrm{X}$ & $\mathrm{x}$ & $\mathrm{x}$ & & $\mathrm{x}$ \\
\hline \multicolumn{7}{|l|}{ Erythrinidae } \\
\hline Hoplias malabaricus (Bloch, 1794) & traíra & $\mathrm{X}$ & & $\mathrm{X}$ & & \\
\hline \multicolumn{7}{|l|}{ Characidae } \\
\hline Astyanax eigenmanniorum (Cope, 1894) & lambari & $\mathrm{X}$ & $\mathrm{x}$ & $\mathrm{X}$ & $\mathrm{X}$ & $\mathrm{X}$ \\
\hline A. aff. fasciatus & lambari & $\mathrm{x}$ & $\mathrm{x}$ & $\mathrm{x}$ & $\mathrm{x}$ & \\
\hline A. jacuhiensis (Cope, 1894) & lambari & $\mathrm{x}$ & $\mathrm{x}$ & $\mathrm{x}$ & $\mathrm{x}$ & $\mathrm{x}$ \\
\hline Astyanax sp. & lambari & $\mathrm{x}$ & & $\mathrm{x}$ & & \\
\hline Bryconamericus iheringii (Boulenger, 1887) & lambari & $\mathrm{x}$ & & $\mathrm{x}$ & & $\mathrm{x}$ \\
\hline Charax stenopterus (Cope, 1894) & lambari & $\mathrm{X}$ & & $\mathrm{X}$ & $\mathrm{x}$ & \\
\hline Cheirodon ibicuhiensis Eigenmann, 1915 & lambari & & $\mathrm{X}$ & $\mathrm{x}$ & & $\mathrm{X}$ \\
\hline C. interruptus (Jenyns, 1842) & lambari & & & $\mathrm{x}$ & & $\mathrm{x}$ \\
\hline Cyanocharax alburnus (Hensel, 1870) & lambari & & & $\mathrm{x}$ & $\mathrm{x}$ & $\mathrm{x}$ \\
\hline Hyphessobrycon anisitsi (Eigenmann, 1907) & lambari & & & $\mathrm{x}$ & & \\
\hline H. bifasciatus Ellis, 1911 & lambari & & & $\mathrm{x}$ & $\mathrm{X}$ & \\
\hline H. luetkenii (Boulenger, 1887) & lambari & $\mathrm{x}$ & $\mathrm{x}$ & $\mathrm{x}$ & $\mathrm{x}$ & $\mathrm{x}$ \\
\hline H. meridionalis Ringuelet, Miquelarena \& Menni, 1978 & lambari & & & $\mathrm{x}$ & $\mathrm{x}$ & $\mathrm{x}$ \\
\hline Macropsobrycon uruguayanae Eigenmann, 1915 & lambari & & $\mathrm{x}$ & $\mathrm{x}$ & $\mathrm{x}$ & \\
\hline Oligosarcus jenynsii (Günther, 1864) & dentuço & $\mathrm{x}$ & $\mathrm{x}$ & $\mathrm{x}$ & & $\mathrm{X}$ \\
\hline O. robustus Menezes, 1969 & dentuço & $\mathrm{x}$ & $\mathrm{x}$ & $\mathrm{x}$ & & \\
\hline \multicolumn{7}{|l|}{ Crenuchidae } \\
\hline Characidium rachovii (Regan, 1913) & lambari & & & $\mathrm{X}$ & $\mathrm{X}$ & \\
\hline C. tenue (Cope, 1894) & lambari & & & $\mathrm{x}$ & & \\
\hline \multicolumn{7}{|l|}{ SILURIFORMES } \\
\hline Auchenipteridae & & & & & & \\
\hline Trachelyopterus lucenai Bertoletti, Pezzi Silva \& Pereira, 1995 & penharol & $\mathrm{X}$ & & & & $\mathrm{X}$ \\
\hline Heptapteridae & & & & & & \\
\hline Pimelodella australis Eigenmann, 1917 & mandi & $\mathrm{X}$ & & $\mathrm{X}$ & & $\mathrm{X}$ \\
\hline Rhamdia aff. quelen & jundiá & $\mathrm{x}$ & & $\mathrm{x}$ & $\mathrm{X}$ & $\mathrm{x}$ \\
\hline Pimelodidae & & & & & & \\
\hline Parapimelodus nigribarbis (Boulenger, 1889) & mandi & $\mathrm{X}$ & & & & \\
\hline Pimelodus maculatus Lacépède, 1803 & pintado & $\mathrm{x}$ & & & & \\
\hline Trychomycteridae & & & & & & \\
\hline Homodiaetus anisitsi Eigenmann \& Ward, 1907 & candiru & & & $\mathrm{X}$ & & \\
\hline Pseudopimelodidae & & & & & & \\
\hline Microglanis cottoides (Boulenger, 1891) & bagrinho & & & $\mathrm{X}$ & & \\
\hline Callichthyidae & & & & & & \\
\hline Corydoras paleatus (Jenyns, 1842) & & & & $\mathrm{x}$ & & \\
\hline Hoplosternum littorale (Hancock, 1828) & tamboatá & $\mathrm{X}$ & & & & \\
\hline Loricariidae & & & & & & \\
\hline Hisonotus taimensis (Buckup, 1981) & limpa-fundo & & & $\mathrm{X}$ & $\mathrm{x}$ & $\mathrm{X}$ \\
\hline Hypostomus commersoni Valenciennes, 1836 & cascudo & $\mathrm{X}$ & & $\mathrm{x}$ & & \\
\hline Loricariichthys anus (Valenciennes, 1836) & cascudo-viola & $\mathrm{x}$ & & $\mathrm{x}$ & & $\mathrm{x}$ \\
\hline Rineloricaria cadeae (Hensel, 1868) & cascudo-viola & & & $\mathrm{x}$ & & \\
\hline Rineloricaria longicauda Reis, 1983 & cascudo-viola & $\mathrm{X}$ & & $\mathrm{x}$ & & $\mathrm{x}$ \\
\hline R. strigilata (Hensel, 1868) & cascudo-viola & $\mathrm{x}$ & & $\mathrm{x}$ & & $\mathrm{x}$ \\
\hline CYPRINODONTIFORMES & & & & & & \\
\hline Anablepidae & & & & & & \\
\hline Jenynsia multidentata (Jenyns, 1842) & barrigudinho & $\mathrm{X}$ & $\mathrm{x}$ & $\mathrm{x}$ & $\mathrm{X}$ & \\
\hline Poeciliidae & & & & & & \\
\hline Cnesterodon decemmaculatus (Jenyns, 1842) & barrigudinho & & $\mathrm{X}$ & $\mathrm{X}$ & $\mathrm{X}$ & \\
\hline Phalloceros caudimaculatus (Hensel, 1868) & barrigudinho & & & $\mathrm{x}$ & $\mathrm{x}$ & \\
\hline ATHERINIFORMES & & & & & & \\
\hline Atherinopsidae & & & & & & \\
\hline Odontesthes bonariensis (Valenciennes, 1835) & peixe-rei & $\mathrm{x}$ & & & & $\mathrm{x}$ \\
\hline O. humensis de Buen, 1953 & peixe-rei & $\mathrm{x}$ & & $\mathrm{x}$ & $\mathrm{X}$ & \\
\hline O. mirinensis Bemvenuti, 1995 & peixe-rei & $\mathrm{x}$ & & $\mathrm{x}$ & & $\mathrm{X}$ \\
\hline O. perugiae Evermann \& Kendall, 1906 & peixe-rei & $\mathrm{x}$ & $\mathrm{X}$ & $\mathrm{x}$ & $\mathrm{x}$ & $\mathrm{x}$ \\
\hline O. retropinnis (de Buen, 1953) & peixe-rei & $\mathrm{x}$ & & $\mathrm{x}$ & & \\
\hline PERCIFORMES & & & & & & \\
\hline Cichlidae & & & & & & \\
\hline Australoheros facetum (Jenyns, 1842) & cará & $\mathrm{X}$ & & $\mathrm{X}$ & $\mathrm{X}$ & \\
\hline Cichlasoma portalegrense (Hensel, 1870) & cará & $\mathrm{x}$ & & & & \\
\hline Crenicichla lepidota Heckel, 1840 & joaninha & $\mathrm{x}$ & & $\mathrm{x}$ & $\mathrm{X}$ & $\mathrm{x}$ \\
\hline C. punctata Hensel, 1870 & joaninha & $\mathrm{x}$ & & $\mathrm{x}$ & & \\
\hline Geophagus brasiliensis (Quoy \& Gaimard, 1824) & cará & $\mathrm{x}$ & $\mathrm{X}$ & $\mathrm{x}$ & & $\mathrm{x}$ \\
\hline Gymnogeophagus gymnogenys (Hensel, 1870) & cará & $\mathrm{x}$ & & $\mathrm{x}$ & & $\mathrm{x}$ \\
\hline G. rhabdotus (Hensel, 1870) & cará & & & $\mathrm{X}$ & & $\mathrm{X}$ \\
\hline Gobiidae & & & & & & \\
\hline Ctenogobius schufeldti (Jordan \& Eigenmann, 1887) & gobídeo & & & $\mathrm{x}$ & & $\mathrm{x}$ \\
\hline
\end{tabular}


Tabela II. Percentual numérico, classificação de abundância e frequencia (4, abundante e frequente; 3, abundante e não-frequente; 2, nãoabundante e frequente; 1 , presente), número de indivíduos (N), comprimento total mínimo (CT Mín.) e máximo (CT Máx.) das espécies de peixes amostradas nas zonas litoral, profunda e limnética da lagoa Mangueira, entre março de 2001 e fevereiro de 2007.

\begin{tabular}{|c|c|c|c|c|c|c|}
\hline \multicolumn{7}{|c|}{ Percentual Numérico } \\
\hline Espécies & Zona Litoral & Zona Profunda & Zona Limnética & $\mathrm{N}$ & CT Mín. & CT Máx. \\
\hline Astyanax eigenmanniorum & $2,2(4)$ & $0,1(1)$ & $9,1(4)$ & 956 & 24 & 214 \\
\hline A. aff. fasciatus & $0,3(1)$ & & $5,2(4)$ & 421 & 26 & 188 \\
\hline A. jacuhiensis & $1,0(2)$ & $0,0(1)$ & $1,0(1)$ & 221 & 36 & 127 \\
\hline Astyanax sp. & $0,0(1)$ & & $2,8(2)$ & 212 & 10 & 150 \\
\hline Australoheros facetum & $0,7(1)$ & & $0,1(1)$ & 103 & 14 & 172 \\
\hline Bryconamericus iheringii & $4,6(4)$ & $0,6(2)$ & $0,8(1)$ & 736 & 22 & 111 \\
\hline Characidium rachovii & $0,0(1)$ & & & 5 & 23 & 74 \\
\hline C. tenue & $0,0(1)$ & & & 3 & 60 & 64 \\
\hline Charax stenopterus & $0,2(1)$ & & $0,6(1)$ & 73 & 24 & 120 \\
\hline Cheirodon ibicuhiensis & $0,1(1)$ & $3,5(2)$ & & 457 & 18 & 58 \\
\hline C. interruptus & $0,2(1)$ & $0,3(1)$ & & 70 & 25 & 63 \\
\hline Cichlasoma portalegrense & & & $0,0(1)$ & 2 & 94 & 119 \\
\hline Cnesterodon decemmaculatus & $0,1(1)$ & & & 16 & 15 & 34 \\
\hline Corydoras paleatus & $0,3(2)$ & & & 39 & 46 & 71 \\
\hline Crenicichla lepidota & $0,3(1)$ & $0,1(1)$ & $0,2(1)$ & 55 & 27 & 200 \\
\hline C. punctata & $2,6(3)$ & & $0,1(1)$ & 358 & 27 & 293 \\
\hline Ctenogobius schufeldti & $0,1(1)$ & $0,4(2)$ & & 58 & 16 & 73 \\
\hline Cyanocharax alburnus & $21,7(4)$ & $69,3(4)$ & & 11613 & 13 & 84 \\
\hline Cyphocharax voga & $0,1(1)$ & $0,0(1)$ & $7,5(4)$ & 584 & 64 & 296 \\
\hline Geophagus brasiliensis & $1,2(2)$ & $0,1(2)$ & $0,0(1)$ & 169 & 22 & 272 \\
\hline Gymnogeophagus gymnogenys & $3,4(4)$ & $0,3(2)$ & $0,0(1)$ & 482 & 21 & 185 \\
\hline G. rhabdotus & $0,0(1)$ & $0,0(1)$ & & 5 & 94 & 140 \\
\hline Hisonotus taimensis & $0,1(1)$ & $0,0(1)$ & & 14 & 20 & 53 \\
\hline Homodiaetus anisitsi & $0,0(1)$ & & & 1 & 30 & 30 \\
\hline Hoplias malabaricus & $0,1(1)$ & & $2,0(2)$ & 147 & 23 & 485 \\
\hline Hoplosternum littorale & & & $0,0(1)$ & 3 & 209 & 216 \\
\hline Hyphessobrycon anisitsi & $0,0(1)$ & & & 1 & 67 & 67 \\
\hline H. bifasciatus & $0,1(1)$ & & & 13 & 28 & 48 \\
\hline H. luetkenii & $4,1(4)$ & $0,1(1)$ & $0,0(1)$ & 552 & 19 & 85 \\
\hline H. meridionalis & $0,0(1)$ & $0,1(1)$ & & 24 & 20 & 45 \\
\hline Hypostomus commersoni & $0,0(1)$ & & $0,0(1)$ & 2 & 220 & 610 \\
\hline Jenynsia multidentata & $29,8(4)$ & & $0,1(1)$ & 3937 & 12 & 170 \\
\hline Loricariichthys anus & $0,0(1)$ & $0,7(2)$ & $0,4(1)$ & 129 & 21 & 458 \\
\hline Macropsobrycon uruguayanae & $0,0(1)$ & & & 4 & 24 & 31 \\
\hline Microglanis cottoides & $0,0(1)$ & & & 1 & 77 & 77 \\
\hline Odontesthes bonariensis & & $0,0(1)$ & $1,7(2)$ & 130 & 165 & 410 \\
\hline O. humensis & $0,2(1)$ & & $2,4(2)$ & 198 & 35 & 377 \\
\hline O. mirinensis & $13,2(4)$ & $0,4(2)$ & $14,1(4)$ & 2878 & 16 & 360 \\
\hline O. perugiae & $0,2(1)$ & $0,2(2)$ & $5,7(4)$ & 476 & 47 & 280 \\
\hline O. retropinnis & $0,0(1)$ & & $0,6(1)$ & 49 & 150 & 331 \\
\hline Odontesthes spp. & $5,9(4)$ & & & 773 & 14 & 124 \\
\hline Oligosarcus jenynsii & $0,4(2)$ & $0,1(1)$ & $41,1(4)$ & 3132 & 12 & 335 \\
\hline$O$. robustus & $0,0(1)$ & & $2,2(2)$ & 169 & 59 & 336 \\
\hline Parapimelodus nigribarbis & & & $0,1(1)$ & 5 & 118 & 242 \\
\hline Phalloceros caudimaculatus & $0,2(1)$ & & & 27 & 18 & 41 \\
\hline Pimelodella australis & $0,0(1)$ & $0,0(1)$ & $0,1(1)$ & 9 & 84 & 149 \\
\hline Pimelodus maculatus & & & $0,0(1)$ & 1 & 263 & 263 \\
\hline Platanichthys platana & $6,4(4)$ & $23,5(4)$ & & 3818 & 15 & 87 \\
\hline Rhamdia aff. quelen & $0,0(1)$ & $0,0(1)$ & $0,1(1)$ & 15 & 224 & 490 \\
\hline Rineloricaria cadeae & $0,0(1)$ & & & 3 & 47 & 75 \\
\hline R. longicauda & $0,1(1)$ & $0,0(1)$ & $0,2(1)$ & 29 & 35 & 175 \\
\hline R. strigilata & $0,0(1)$ & $0,0(1)$ & $0,0(1)$ & 5 & 75 & 203 \\
\hline Trachelyopterus lucenai & & $0,0(1)$ & $1,7(2)$ & 120 & 153 & 218 \\
\hline $\mathrm{N}$ total & 13213 & 12619 & 7471 & 33303 & & \\
\hline
\end{tabular}

$\mathrm{O}$ valor da similaridade em termos de presença/ ausência de espécies medido pelo coeficiente de Jaccard foi de 0,52 ; resultando em padrões de dominância diferenciados e, consequentemente, em baixa similaridade em termos de abundância relativa $(\mathrm{P}=33,79)$ (Tab. III). Também sazonalmente as assembleias foram pouco similares entre si. Pequena variabilidade nos padrões de dominância foi verificada no outono no ponto norte, e no inverno e na primavera no ponto sul (Tab. IV).

A análise do padrão geral de abundância por classe de CT mostrou que nas zonas litoral e profunda mais de
$50 \%$ dos indivíduos capturados apresentavam entre 25 e $50 \mathrm{~mm}$ de CT, enquanto que os indivíduos pelágicos, embora tenham apresentado maior variação de tamanhos, foram maiores, com moda em $160 \mathrm{~mm}$ CT (Fig. 4).

Os resultados de abundancia relativa por classe de CT dos indivíduos coletados na zona litoral dos pontos de amostragem ao norte e ao sul da lagoa (PELD-CNPq site 7) mostraram modelos diferenciados de estrutura de tamanhos das assembleias. Enquanto no ponto norte $65 \%$ dos indivíduos apresentou tamanhos entre 25 e $50 \mathrm{~mm}$, no ponto sul $90 \%$ dos indivíduos apresentou esta variação (Figs. 5, 6). 


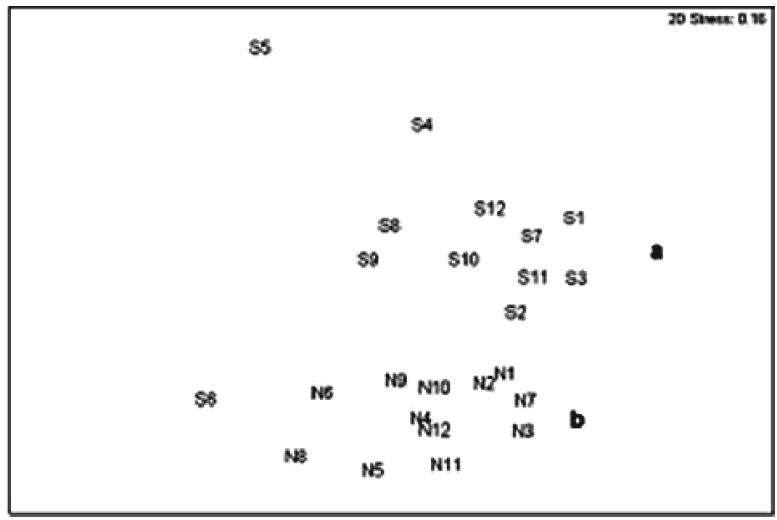

Figura 3. Ordenação pelo método MDS a partir dos dados de presença/ ausência das espécies de peixes coletadas mensalmente entre março de 2004 e fevereiro de $2005 \mathrm{com}$ rede de arrasto de praia, nos pontos de amostragem do programa PELD-site 7 na zona litoral da lagoa Mangueira (N, norte; S, sul; 1 a 12, amostras). Grupos delineados no nível de $50 \%$ de similaridade em destaque (a e b). Estresse da ordenação MDS $=0,16$.

Tabela III. Importância relativa baseada em frequencia de ocorrência e percentual numérico dos peixes coletados na zona litoral da lagoa Mangueira entre março de 2004 e fevereiro de 2005, com rede de arrasto, nos pontos de amostragem do programa PELD-site 7 (dados parcialmente publicados em GARCIA et al., 2006) (4, abundante e frequente; 3 , abundante e não-frequente; 2, não-abundante e frequente; 1 , presente; vazio, ausência), número de indivíduos coletados $(\mathrm{N})$, número de espécies $(\mathrm{S})$.

\begin{tabular}{|c|c|c|}
\hline & Importânc & Relativa \\
\hline Espécies & Norte & Sul \\
\hline Astyanax aff. fasciatus & 1 & 1 \\
\hline A. eigenmanniorum & 2 & 4 \\
\hline A. jacuhiensis & 2 & 1 \\
\hline Astyanax sp. & 1 & 1 \\
\hline Australoheros facetum & & 1 \\
\hline Bryconamericus iheringii & 1 & 4 \\
\hline Charax stenopterus & 1 & 1 \\
\hline Cheirodon ibicuhiensis & 1 & \\
\hline C. interruptus & 1 & 1 \\
\hline Cnesterodon decemmaculatus & & 1 \\
\hline Corydoras paleatus & & 2 \\
\hline Crenicichla lepidota & & 2 \\
\hline C.a punctata & & 1 \\
\hline Ctenogobius schufeldti & & 1 \\
\hline Cyanocharax alburnus & 4 & 4 \\
\hline Cyphocharax voga & 1 & 1 \\
\hline Geophagus brasiliensis & 1 & 2 \\
\hline Gymnogeophagus gymnogenys & & 4 \\
\hline G. rhabdotus & 1 & 1 \\
\hline Hoplias malabaricus & 1 & 1 \\
\hline Hyphessobrycon anisitsi & 1 & \\
\hline H. bifasciatus & & 1 \\
\hline H. luetkenii & 2 & 4 \\
\hline Hypostomus commersoni & 1 & \\
\hline Jenynsia multidentata & 4 & 4 \\
\hline Loricariichthys anus & & 1 \\
\hline Macropsobrycon uruguayanae & & 1 \\
\hline Microglanis cottoides & 1 & \\
\hline Odontesthes humensis & 1 & 1 \\
\hline O. mirinensis & 4 & 1 \\
\hline O. perugiae & 1 & 1 \\
\hline O. retropinnis & 1 & \\
\hline Odontesthes spp. & 4 & 2 \\
\hline Oligosarcus jenynsii & 2 & 1 \\
\hline Phalloceros caudimaculatus & & 1 \\
\hline Pimelodella australis & & 1 \\
\hline Platanichthys platana & 4 & 4 \\
\hline Rhamdia aff. quelen & 1 & \\
\hline Rineloricaria cadeae & & 1 \\
\hline R. longicauda & 1 & 1 \\
\hline$N(S)$ & $4198(26)$ & $2799(33)$ \\
\hline Esforço & 60 & 60 \\
\hline
\end{tabular}

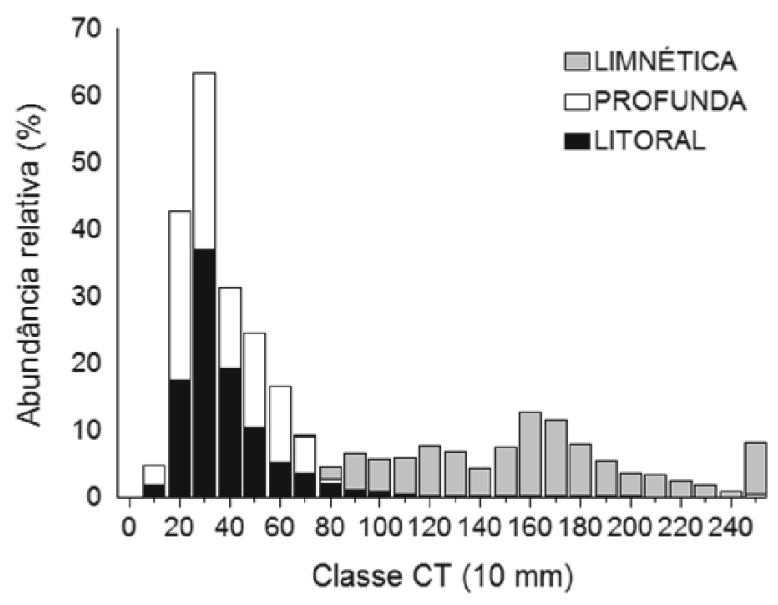

Figura 4. Abundância relativa por classe de comprimento total (CT) de peixes coletados entre março de 2001 e fevereiro de 2007 nas zonas litoral, profunda e limnética costeira da lagoa Mangueira.

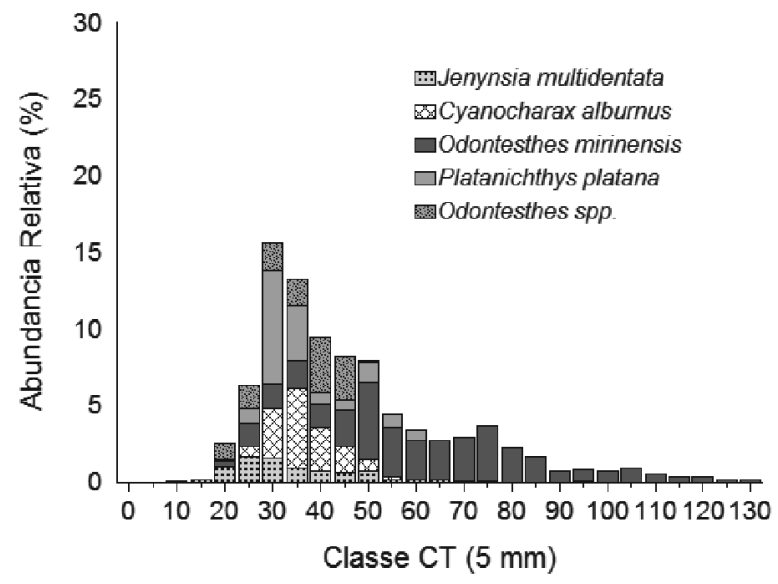

Figura 5. Abundância relativa por classe de comprimento total (CT) das espécies de peixes dominantes coletadas entre março de 2004 e fevereiro de 2005 nos pontos de amostragem ao norte (PELD-CNpq site 7) da zona litoral da lagoa Mangueira.

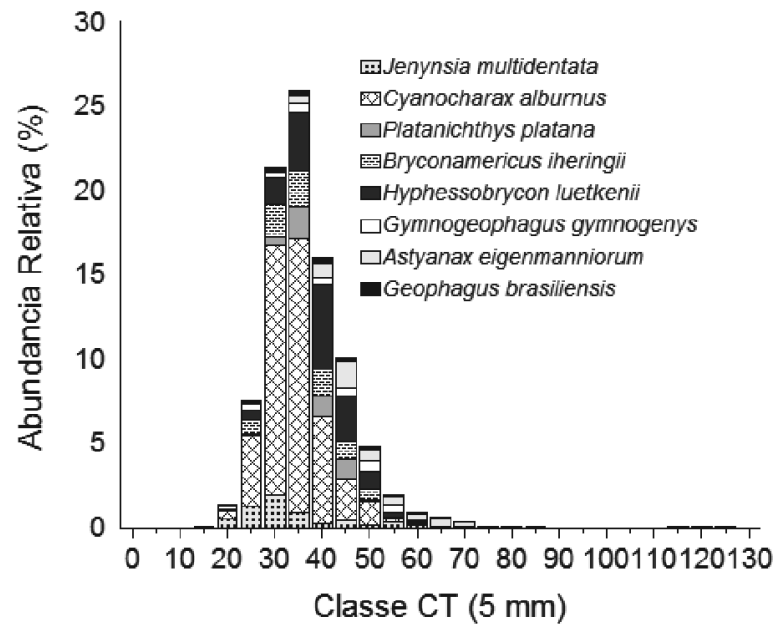

Figura 6. Abundância relativa por classe de comprimento total (CT) das espécies de peixes dominantes coletadas entre março de 2004 e fevereiro de 2005 nos pontos de amostragem ao sul (PELDCNpq site 7) da zona litoral da lagoa Mangueira. 
Tabela IV. Variação sazonal da importância relativa (4, abundante e frequente; 3, abundante e não-frequente; 2, não-abundante e frequente; 1, presente; vazio, ausência) e valores de CPUE das principais espécies (no mínimo frequentes) de peixes coletadas nos pontos de amostragem do programa PELD-site 7 na zona litoral da lagoa Mangueira entre março de 2004 e fevereiro de 2005 , com rede de arrasto de praia. Número de indivíduos coletados $(\mathrm{N})$, número de espécies $(\mathrm{S})$, coeficiente de Jaccard $(\mathrm{Cj})$, percentual de similaridade mínima $(\mathrm{P})$.

\begin{tabular}{|c|c|c|c|c|c|c|c|c|}
\hline \multirow[b]{2}{*}{ Espécies } & \multicolumn{2}{|c|}{ Verão } & \multicolumn{2}{|c|}{ Outono } & \multicolumn{2}{|c|}{ Inverno } & \multicolumn{2}{|c|}{ Primavera } \\
\hline & Norte & Sul & Norte & Sul & Norte & Sul & Norte & Sul \\
\hline Astyanax eigenmanniorum & $1,3(2)$ & $5,7(4)$ & $3,4(4)$ & $3,5(2)$ & $0,1(1)$ & $0,3(4)$ & $0,4(1)$ & $0,5(1)$ \\
\hline Astyanax jacuhiensis & $4,4(2)$ & $0,2(1)$ & $1,7(2)$ & $0,4(1)$ & $0,4(2)$ & & $0,1(1)$ & \\
\hline Bryconamericus iheringii & $0,5(1)$ & $3,8(4)$ & & $10,1(4)$ & $0,3(1)$ & $0,4(4)$ & & $1,6(3)$ \\
\hline Charax stenopterus & $0,9(1)$ & $0,7(1)$ & & $0,2(1)$ & & $0,7(1)$ & & $0,5(2)$ \\
\hline Cheirodon interruptus & $0,3(1)$ & & & & & & $0,4(2)$ & $0,5(1)$ \\
\hline Crenicichla lepidota & & $1,2(2)$ & & $0,7(1)$ & & $0,7(1)$ & & $0,4(2)$ \\
\hline Ctenogobius schufeldti & & $0,1(1)$ & & $0,5(2)$ & & & & \\
\hline Cyanocharax alburnus & $21,9(4)$ & $39,7(4)$ & $11,4(4)$ & $4,9(4)$ & $1,9(2)$ & $0,3(4)$ & $5,9(4)$ & $4,9(4)$ \\
\hline Geophagus brasiliensis & & $1,6(2)$ & $0,8(1)$ & $1,7(2)$ & & $0,9(4)$ & & $0,9(2)$ \\
\hline Gymnogeophagus gymnogenys & & $3,5(4)$ & & $2,8(2)$ & & $0,2(2)$ & & $0,2(1)$ \\
\hline Hoplias malabaricus & & $0,7(1)$ & & & & & $0,1(1)$ & $0,3(2)$ \\
\hline Hyphessobrycon luetkenii & $0,1(1)$ & $7,5(4)$ & $5,7(4)$ & $4,9(4)$ & $0,8(2)$ & $0,5(4)$ & $0,3(1)$ & $14,7(4)$ \\
\hline Jenynsia multidentata & $18,9(4)$ & $6,0(4)$ & $1,3(2)$ & $1,7(2)$ & $0,5(2)$ & $0,2(2)$ & $1,3(2)$ & $3,4(4)$ \\
\hline Odontesthes mirinensis & $31,1(4)$ & & $9,6(4)$ & & $26,9(4)$ & $0,2(2)$ & $39,5(4)$ & \\
\hline Odontesthes spp. & $21,6(4)$ & & & & & $0,3(4)$ & $14,0(4)$ & $1,9(4)$ \\
\hline Oligosarcus jenynsii & $0,7(1)$ & & $0,3(1)$ & $1,0(1)$ & $0,1(1)$ & & $0,7(2)$ & $0,4(2)$ \\
\hline Platanichthys platana & $33,5(4)$ & $0,3(2)$ & $1,3(2)$ & $9,7(4)$ & $1,3(2)$ & & $9,7(4)$ & $0,7(1)$ \\
\hline $\mathrm{N}$ & 2029 & 1085 & 567 & 1168 & 508 & 59 & 1094 & 487 \\
\hline S & 16 & 21 & 16 & 22 & 16 & 15 & 13 & 20 \\
\hline $\mathrm{C}$ & 0,31 & & 0,41 & & 0,23 & & 0,40 & \\
\hline$P^{j}$ & & & & & & & & \\
\hline
\end{tabular}

\section{DISCUSSÃO}

O primeiro ponto importante a ser considerado no estudo da assembleia de peixes é a obtenção de uma lista de espécies (Schifino et al., 2004). Estudos conduzidos com o objetivo de obter listas de espécies (BuckuP \& Malabarba, 1983), padrões de distribuição (Grosser et al., 1994) e dominância (GARCIA et al., 2006) foram realizados para a EETaim, da qual a porção norte da lagoa Mangueira faz parte. Buckup \& Malabarba (1983) coletaram e identificaram 53 espécies de peixes, embora não tenham feito referência ao local exato de coleta. Grosser et al. (1994) identificaram 51 espécies, sendo 37 destas associadas à lagoa Mangueira. GARCIA et al. (2006) registraram a presença de 62 espécies de peixes para a porção norte da lagoa Mangueira e apresentaram, uma lista comparada de espécies e uma descrição preliminar dos padrões de dominância nas principais lagoas que compõem o sistema. Portanto, as 52 espécies de peixes identificadas no presente estudo corresponderam a 83,9 $\%$ das espécies descritas para EETaim, revelando uma alta diversidade de peixes para a lagoa Mangueira.

A importância da lagoa Mangueira em riqueza de espécies fica evidente tanto quando comparada ao número total de espécies válidas para o sistema da laguna dos Patos (148) (Malabarba, 2008), abrangendo $35 \%$ destas, quanto quando comparada a outras lagoas costeiras do RS. MALABarba \& IsAiA (1992) identificaram 57 espécies nas lagoas costeiras do litoral norte do Estado, enquanto ScHIFINo et al. (2004) identificaram 22 espécies para a lagoa Fortaleza $\left(19,06 \mathrm{~km}^{2}\right)$, também na porção norte do RS.

Em termos de composição específica de grupos, a maior proporção de espécies das ordens Characiformes e Siluriformes segue uma tendência observada nos ambientes aquáticos continentais da região neotropical (Castro \& Menezes, 1998; Lowe-McConnell, 1999; Shibatta et al., 2002; Schifino et al., 2004). Destaca-se, entretanto, o alto número de espécies da família Atherinopsidae na lagoa Mangueira, com a ocorrência de cinco das sete espécies de peixes-rei descritas para a bacia da laguna dos Patos (Malabarba, 2008).

As amostras conduzidas com rede de arrasto de praia nas margens rasas da zona litoral e as amostras de rede de emalhe nas áreas abertas da zona limnética resultaram em padrões diferenciados de dominância de espécies. Uma assembleia mais rica em espécies, porém de indivíduos de menor tamanho ( $<75 \mathrm{~mm})$, constituída principalmente por lambaris (família Characidae), a sardinha (Platanichthys platana (Regan, 1917)) e os juvenis de peixes-rei (Odontesthes spp.), dominou as margens rasas, enquanto as zonas limnéticas foram menos ricas em espécies, porém dominadas por peixes de maior tamanho (>90 mm), tais como o birú (Cyphocharax voga (Hensel, 1870)), os grandes caracídeos, adultos dos peixes-rei, a traíra (Hoplias malabaricus (Bloch, 1794)) e o penharol (Trachelyopterus lucenai Bertoletti, Pezzi Silva \& Pereira 1995). Este padrão de pequenos peixes habitando áreas rasas protegidas vs. grandes peixes encontrados em regiões limnéticas e profundas é comum em outros ambientes aquáticos continentais, tais como os estuários (e. g. o estuário da laguna dos Patos, VIEIRA et al., 1998, já tendo sido descrito para os banhados de água doce da EETaim (GARCIA et al., 2006).

Existem, entretanto, diferenças substanciais entre os amostradores utilizados no presente trabalho. As redes 
de arrasto de praia e de fundo são artefatos de pesca ativos, mais apropriados à captura de pequenos peixes, enquanto as redes de emalhe são artefatos passivos, mais apropriados à captura de peixes grandes, de natação rápida e que habitam a meia água (HAYES, 1989; RoZAS \& Minello, 1997). Outro fator importante é o tamanho da malha utilizada que, nesse caso, foi igual para as redes de arrasto de praia e fundo (mínimo $5 \mathrm{~mm}$ ) e bem maior nas redes de emalhe e tarrafa (mínimo $15 \mathrm{~mm}$ ).

Neste sentido, a ausência, nas coletas da zona limnética, de Cyanocharax alburnus (Hensel, 1870) e Platanichthys platana, duas espécies de pequeno porte e que são dominantes nos arrastos de fundo e praia, poderia estar relacionada ao tamanho de malha ou a um hábito exclusivamente demersal, uma vez que estas espécies foram abundantes nas zonas rasas e no fundo, com médias de tamanhos semelhantes. Muitas espécies apresentam tamanho diminuto não sendo capturadas pelas redes de emalhe, o que não significa, necessariamente, que estejam ausentes na zona limnética. A ausência sistemática de $C$. alburnus e $P$. platana em ambos os amostradores de meia-água (rede de emalhe e tarrafa) sugere, contudo, que estas espécies evitam a região limnética aberta.

Os resultados nos padrões de dominância nas três unidades de hábitat analisadas indicam uma equidade em relação à percentagem de espécies dominantes entre as assembleias das zonas rasas (marginais) e abertas (limnéticas). Comparações com a zona profunda, neste caso, são pouco apropriadas devido ao reduzido esforço amostral.

Em relação aos resultados obtidos por GARCIA et al. (2006), o presente estudo mostrou maior riqueza de espécies, principalmente nas amostragens da zona litoral (de 26 para 46 espécies). A captura das duas espécies Hyphessobrycon meridionalis Ringuelet, Miquelarena \& Menni, 1978 e Hisonotus taimensis (Buckup, 1981) é atribuída às amostragens na porção central da lagoa, enquanto a captura das demais, às amostragens na porção sul. As espécies Rineloricaria strigilata (Hensel, 1868) e Characidium tenue (Cope, 1894) são registradas pela primeira vez para o sistema Taim-lagoa Mangueira. A ocorrência destas espécies na lagoa Mangueira, no entanto, sugere que as mesmas também ocorram na área da reserva ecológica.

As diferenças observadas na riqueza, composição e padrões de dominância das espécies da assembleia da zona litoral entre os pontos norte e sul da lagoa Mangueira podem estar relacionadas à heterogeneidade na estrutura do hábitat. $\mathrm{O}$ ponto norte constitui-se de uma praia aberta, mais exposta à ação dos ventos, de baixo declive e profundidade, que oferece poucos espaços seguros para os peixes, enquanto o ponto sul é mais protegido, profundo e densamente colonizado por macrófitas submersas. Ainda que dados quantitativos referentes à cobertura de macrófitas não tenham sido medidos, sugere-se, como hipótese, que a maior profundidade no ponto sul possa favorecer o crescimento da vegetação submersa, em razão da baixa hidrodinâmica, ressuspensão de sedimentos e, consequentemente, turbidez da água (ver FERNÁNDEZ et al., 1998 para mais detalhes).
Bancos de macrófitas submersas são importantes habitats para muitos grupos de organismos (LALONDE \& Downing, 1992; Albertoni \& Würdig, 1996; Agostinho et al., 2003) que utilizam as plantas como substrato, refúgio, local de alimentação ou como uma fonte direta de nutrientes (CHERUVELIL et al., 2000; BuRKs et al., 2001; MARKLUnd et al., 2001). Este elevado suprimento alimentar tem sido descrito como um importante fator para explicar a agregação de peixes em estandes de macrófitas submersas (RoZAS \& ODUM, 1988; GARCIA \& VIEIRA, 1997; Casatti et al., 2003; Pelicice \& Agostinho, 2006; Pelicice et al., 2008) e pode estar relacionado à maior riqueza e número de espécies dominantes observados no ponto sul da lagoa Mangueira. Essa riqueza em espécies fica ainda mais evidente quando comparados os números totais de indivíduos coletados.

A maior abundancia de peixes de menor tamanho e o recrutamento dos juvenis de peixes-rei (Odontesthes spp.) antes da primavera, no ponto sul da lagoa, sugere que este seja um local preferencial para a reprodução, talvez por exibir melhores condições para a sobrevivência e crescimento dos filhotes. Em lagos de regiões temperadas, as funções de área de desova e refúgio de margens vegetadas já foram testadas (RozAs \& ODuM, 1988; ChICK \& McIvor, 1997; Gosch et al., 2006). Contudo, em regiões tropicais, a função de "berçário" da zona litoral é mais conhecida para planícies de inundação sazonais (Welcomme, 1985; Agostinho et al., 2003) e estuários (Silva, 1982; VieIRA \& ScAlabrin, 1991; Garcia \& VieIRA, 1997; Vieira et al., 1998). Em lagos, a estrutura de tamanhos das assembleias de peixes da zona litoral tem sido bem menos estudada (VONO \& BARBOSA, 2001; Schifino et al., 2004; GARCIA et al., 2006), e ainda menos conhecido são as preferências de hábitat dos peixes e suas variações sazonais.

Mesmo considerando as diferenças no esforço amostral e nos amostradores utilizados, padrões diferenciados de composição, dominância e estrutura de tamanhos das assembleias de peixes das zonas litoral, profunda e limnética parecem ocorrer na lagoa Mangueira.

Contudo, a realização de novos estudos, a utilização de novos amostradores e a padronização do esforço amostral juntamente com a manutenção do programa PELD poderão obter evidências mais conclusivas a respeito dos padrões de distribuição e dominância da assembleia de peixes da lagoa Mangueira; trazer valiosas informações sobre a biologia básica das espécies ocorrentes na lagoa, principalmente as de interesse comercial, e subsidiar de forma mais eficiente a projetos de manejo e/ou monitoramento, contribuindo de forma mais efetiva na preservação e/ou conservação do ecossistema da lagoa Mangueira.

Agradecimentos. Aos colegas da FURG que participaram das coletas, triagens e identificação das espécies, em especial a Mário V. Condini, Alex Moresco e Marcelo Burns. Aos Drs. Mónica S. Rodriguez e Luiz R. Malabarba pela ajuda na identificação de espécies e sugestões no manuscrito. Ao Dr. David M. L. M. Marques, ao técnico Antônio Bueno (IPH-UFRGS) e ao Instituto Brasileiro de Meio Ambiente (IBAMA) pelo apoio nas saídas a campo. Este trabalho recebeu apoio financeiro da Coordenadoria de Aperfeiçoamento de Pessoal de Nível Superior (CAPES), do Conselho Nacional de Desenvolvimento Científico e Tecnológico $(\mathrm{CNPq})$ e do programa de Pesquisas Ecológicas de Longa Duração (PELD). 


\section{REFERÊNCIAS BIBLIOGRÁFICAS}

Agostinho, A. A.; Gomes, L. C. \& Julio JR., H. F. 2003. Relações entre macrófitas e fauna de peixes. In: Thomaz, S. M. \& Bini, L. M. eds. Ecologia e manejo de macrófitas aquáticas. Maringá, EDUEM. p.261-279.

Albertoni, E. F. \& Würdig, N. L. 1996. Comunidade de ostracodes associada à macrófitas aquáticas na Lagoa do Gentil, Tramandaí, RS. Acta Limnologica Brasiliensis 8:103-114.

Bemvenuti, M. DE A. 2002. Diferenciação morfológica das espécies de peixes-rei, Odontesthes Evermann \& Kendall (Osteichthyes, Atherinopsidae) no extremo sul do Brasil: morfometria multivariada. Revista Brasileira de Zoologia 19(1):251-287.

Bemvenuti, M. De A. \& Moresco, A. 2005. Peixes: áreas de banhados e lagoas costeiras do Extremo Sul do Brasil. Porto Alegre, ABRH. 63p.

Bemvenuti, M. De A. \& Rodrigues, F. L. 2002. Análise comparativa entre técnicas morfométricas aplicadas a Odontesthes bonariensis (Valenciennes) e Odontesthes humensis De Buen (Osteichthyes, Atherinopsidae). Revista Brasileira de Zoologia 19(3):789-796.

Bruton, M. N. \& Merron, G. S. 1990. The proportion of different eco-ethological sections of reproductive guilds of fishes in some African inland waters. Environmental Biology of Fishes 28:179-187.

Buckup, P. A. 1981. Microlepidogaster taimensis sp. n., novo Hypoptopomatinae da Estação Ecológica do Taim, Rio Grande do Sul, Brasil (Ostariophysi, Loricariidae). Iheringia, Série Zoologia 3:531-548.

Buckup, P. A. \& Malabarba, L. M. 1983. A list of the fishes of the Taim Ecological Station, Rio Grande do Sul, Brazil. Iheringia, Série Zoologia, 63:103-113.

Buckup, P. A. \& ReIs, R. E. 1997. Characidiin Genus Characidium (Teleostei, Characiformes) in southern Brazil, with description of three new species. Copeia 3:531-548.

Burks, R. L.; Jeppesen, E. \& Lodge, D. M. 2001. Littoral zone structures as Daphnia refugia against fish predators. Limnology and Oceanography 46:230-237.

Carpenter, S. R.; Kitchell, J. F. \& Hodgson, J. R. 1985. Cascading trophic interactions and lake productivity. BioScience 25:634-639.

Casatti, L.; Mendes, H. F. \& Ferreira, K. M. 2003. Aquatic macrophytes as feeding site for small fishes in the Rosana reservoir, Paranapanema River, southeastern Brazil. Brazilian Journal of Biology 63:213-222.

Castro, R. M. C. \& Menezes, N. A. 1998. Estudo diagnóstico da diversidade de peixes do Estado de São Paulo. In: CAstro, R. M. C. ed. Biodiversidade do Estado de São Paulo, Brasil: Síntese do conhecimento ao final do século $\mathrm{XX}$, 6: vertebrados. São Paulo, WinnerGraph. p.1-13.

Cheruvelil, K. S.; Soranno, P. A. \& Serbin, R. D. 2000. Macroinvertebrates associated with submerged macrophytes: sample size and power to detect effects. Hydrobiologia 441:133-139

Chick, J. H. \& McIvor, C. C. 1997. Habitat selection by three littoral zone fishes: effects of predation pressure, plant density and macrophyte type. Ecology of Freshwater Fish 6:27-35.

DeAngelis, D. L.; Trexler, J. C. \& Loftus, W. F. 2005. Life history trade-offs and community dynamics of small fishes in a seasonally pulsed wetland. Canadian Journal of Fisheries and Aquatic Science 62:781-790.

Delaney, P. J. V. 1965. Fisiografia e geologia da superfície da Planície Costeira do Rio Grande do Sul. Publicação Especial da Escola de Geologia UFRGS 6:1-195.

Esteves, F. A. 1998. Lagoas Costeiras: Origem, Funcionamento e possibilidades de Manejo. In: Esteves, F. A. ed. Ecologia das lagoas costeiras do Parque Nacional da Restinga de Jurubatiba e do Município de Macaé (RJ). Rio de Janeiro, NUPEM. p.63-87.

Fernández, O. A.; Murphy, K. J.; López Cazorla, A.; Sabbatini, M. R.; Lazzari, M. A.; Domaniewski, J. C. J. \& Irigoyen, J. H. 1998. Interrelationships of fish and channel environmental conditions with aquatic macrophytes in an Argentine irrigation system. Hydrobiologia 380:15-25.
Garcia, A. M. \& Vieira, J. P. 1997. Abundância e diversidade da assembléia de peixes dentro e fora de uma pradaria de Ruppia maritima L., no estuário da Lagoa dos Patos (RS-Brasil). Revista Atlântica 19:161-181.

Garcia, A. M.; Vieira, J. P.; Bemvenuti, M. A.; Motta Marques, D. M. L.; Burns, M.; Moresco, A. \& Condini, V. 2006. Checklist comparison and dominance patterns of the fauna at Taim Wetland, South Brazil. Neotropical Icththyology 4(2):261268.

GAUCH JR., H. G. 1982. Multivariate analysis in community ecology. London, Cambridge University. 298p.

Gosch, N. J. C.; Phelps, Q. E.; Willis, D. W. 2006. Habitat characteristics at bluegill spawning colonies in a South Dakota glacial lake. Ecology of Freshwater Fish 15:464-469.

Grosser, K. M.; Koch, W. R. \& DrugG-Hahn, S. 1994. Ocorrência e distribuição de peixes na estação ecológica do Taim, Rio Grande do Sul, Brasil, (Pisces, Teleostomi). Iheringia, Série Zoologia 77:89-98.

Hayes, J. W. 1989. Comparison between a fine mesh trap net and five other fishing gears for sampling shallow-lake fish communities in New Zealand. New Zealand Journal of Marine and Freshwater Research 23:321-324.

Krebs, C. J. 1989. Ecological Metodology. New York, Harper \& Row. 653p.

Lalonde, S. \& Downing, J. A. 1992. Phytofauna of eleven macrophyte beds of differing trophic status, depth, and composition. Canadian Journal of Fisheries and Aquatic Sciences 49: 992-1000.

Lowe-McConnell, R. H. 1999. Estudos ecológicos em comunidades de peixes tropicais. São Paulo, EDUSP. 534p.

Magurran, A. E. 1988. Ecological diversity and its measurement. Princeton, Princeton University. 179p.

Malabarba, L. R. 2008. Catálogo dos peixes de água doce da laguna dos Patos. Disponível em: <http://www.ufrgs.br/ictio/ lagunapatos/>. Acesso em: 23.10.2008.

Malabarba, L. R. \& Isaia, E. A. 1992. The freshwater fish fauna of the rio Tramandaí drainage, Rio Grande do Sul, with a discussion of its local origin. Comunicações do Museu de Ciências da PUCRS, série Zoologia, 5:197-223.

Marklund, O.; Blindow, I. \& Hargeby, A. 2001. Distribution and diel migration of macroinvertebrates within dense submerged vegetation. Freshwater Biology 46:913-924.

Moresco, A. \& Bemvenuti, M. DE A. 2005. Morphologic features and feeding analysis of the black catfish Trachelyopterus lucenai Bertoletti, Pezzi da Silva \& Pereira (Siluriformes, Auchenipteridae). Acta Limnologia Brasilensia 17(1):3744.

Pelicice, F. M. \& Agostinho, A. A. 2006. Feeding ecology of fishes associated with Egeria spp. patches in a tropical reservoir, Brazil. Ecology of Freshwater Fish 15:10-19.

Pelicice, F. M.; Thomaz, S. M. \& Agostinho, A. A. 2008. Simple relationships to predict attributes of fish assemblages in patches of submerged macrophytes. Neotropical Ichthyology 6(4):543-550.

Piedras, S. R. N. \& Pouey, J. L. O. F. 2005. Alimentação do peixerei (Odontesthes bonariensis, Atherinopsidae) nas lagoas Mirim e Mangueira, Rio Grande do Sul, Brasil. Iheringia, Série Zoologia, 95(2):117-120.

ReIs, R. E. 1983. Rineloricaria longicauda e Rineloricaria quadrensis, duas novas espécies de Loricariinae do sul do Brasil (Pisces, Siluriformes, Loricariinae). Iheringia, Série Zoologia, 62:61-80.

Rodrigues, F. L. \& Bemvenuti, M. DE A. 1997. Hábito alimentar e osteologia da boca do peixe-rei, Odontesthes humensis de Buen (Atheriniformes, Atherinopsidae) na Lagoa Mirim, Rio Grande do Sul, Brasil. Revista Brasileira de Zoologia 18(3):793802 .

Rozas, L. P. \& Minello, T. J. 1997. Estimating densities of small fishes and decapod crustaceans in shalow estuarine habitats: a review of sampling design with focus on gear selection. Estuaries 20:199-213.

Rozas, L. P. \& Odum, W. E. 1988. Occupation of submerged aquatic vegetation by fishes: testing the roles of food and refuge. Oecologia 77:101-106.

SchäFER, A. 1984. Fundamentos de ecologia e biogeografia de águas continentais. Porto Alegre, UFRGS. 532p. 
Schifino, L. C.; Fialho, C. B. \& Verani, J. R. 2004. Fish Community Composition, Seasonality and Abundance in Fortaleza Lake, Cidreira. Brazilian Archives of Biology and Technology 47(5):755-763.

Shibatta, O. A.; Orsi, M. L.; Bennemann, S. T. \& Silva-Souza, A. T. 2002. Diversidade e distribuição de peixes na bacia do rio Tibagi. In: Medri, M. E.; Bianchini, E.; Shibatta, O. A. \& Pimenta, J. A. eds. A bacia do rio Tibagi. Londrina, M. E. Medri. v.1. p.403-423.

Silva, C. P. DA. 1982. Ocorrência, distribuição e abundância de peixes na região estuarina de Tramandaí, Rio Grande do Sul. Atlântica 5(1):49-66.

Tomazelli, L. J. \& Villwock, J. A. 2005. Mapeamento Geológico de Planícies Costeiras: O Exemplo da Costa do Rio Grande do Sul. Gravel 3:109-115.

VIEIRA, J. P. 2006. Ecological analogies between estuarine bottom traw fish assemblages from Patos Lake, Rio Grande do Sul, Brazil and York River, Virginia, USA. Revista Brasileira de Zoologia 23(1):234-247.
Vieira, J. P.; Castello, J. P. \& Pereira, L. E. 1998. Ictiofauna. IN Seeliger, U.; Odebrecht, C. \& Castello, J. P. eds. Os ecossistemas costeiro e marinho do extremo sul do Brasil. Rio Grande, Ecoscientia. p.60-68.

Vieira, J. P.; Giarrizzo, T. \& Spach, H. 2006. Nécton. In: Lana, P.; Bianchini, A.; Ribeiro, C.; Niencheski, L.; Filmann, G. \& Santos, C. eds. Avaliação Ambiental de Estuários Brasileiros: Diretrizes Metodológicas. Rio de Janeiro, Museu Nacional. v.1. p.145-147.

VIEIRA, J. P. \& SCALABRin, C. 1991. Migração reprodutiva da "tainha" (Mugil platanus Gunther, 1980) no sul do Brasil. Atlântica 13(1):131-141.

Vono, V. \& Barbosa, F. A. R. 2001. Habitats and littoral zone fish community structure of two natural lakes in southeast Brazil. Environmental Biology of Fishes 61:371-379.

Welcomme, R. L. 1979. Fisheries ecology of floodplain rivers. London, Longman. 317p.

1985. River fisheries. Rome, FAO Fisheries Technical Paper. 330p. 